\title{
Durability of Replicated Distributed Storage Systems
}

\author{
Sriram Ramabhadran and Joseph Pasquale \\ Department of Computer Science \& Engineering \\ University of California, San Diego \\ 9500 Gilman Drive, Mail Code 0404 \\ La Jolla, CA 92093-0404, USA \\ sriram@cs.ucsd.edu, pasquale@cs.ucsd.edu
}

\begin{abstract}
We study the problem of guaranteeing data durability [2] in distributed storage systems based on replication. Our work is motivated by several several recent efforts $[3,5,1]$ to build such systems in a peer-to-peer environment. The key features of this environment which make achieving durability difficult are (1) data lifetimes may be several orders of magnitude larger than the lifetimes of individual storage units, and (2) the system may have little or no control over the participation of these storage units in the system. We use a model-based approach to develop engineering principles for designing automated replication and repair mechanisms to implement durability in such systems.
\end{abstract}

Categories and Subject Descriptors:

D.4.3 File Systems Management: Distributed file systems E.5 Files: Backup / recovery.

General Terms: Design, Performance, Reliability.

Keywords: Replication, Durability.

\section{SYSTEM MODEL}

The system consists of some population of storage nodes on which it can replicate objects. By the term system, we mean the distributed logic whose function is to ensure the durability of the objects it stores. A node may "leave," i.e., no longer be part of, the system at any time, at which point the system cannot retrieve any objects stored on that particular node. Nodes that leave the system are replaced by new ones; thus, we assume that the system is never limited by the inability to find a node on which to replicate. However, it must take measures to ensure that objects persist over a constantly changing population of nodes.

Node model A node participates in the system for some duration of time called its lifetime. This is the time that elapses between the instant a node initially "joins," i.e., becomes part of, the system, and the instant the node permanently leaves it. The lifetime of a node consists of alternating periods of availability and unavailability. Node uptime refers to periods when the node is online (or available), while downtime refers to periods when the node is offline (or unavailable).

Our model for node behavior is based on on the assumptions that node lifetimes, uptimes and downtimes are all exponentially distributed with means $T, t$ and $\bar{t}$ respectively,

Copyright is held by the author/owner.

SIGMETRICS'08, June 2-6, 2008, Annapolis, Maryland, USA.

ACM 978-1-60558-005-0/08/06. where $T \gg t, \bar{t}$. This implies that the underlying random process is Markovian; consequently, we model node behavior by a continuous Markov chain with three states. A node may be either online (state 1), offline (state 2) or dead (state 3). A node always begins its existence in state 1 , alternates between state 1 and state 2 , before it is finally absorbed in state 3 . The transition rates between states can be computed in terms of $T, t$ and $\bar{t}$ as $\lambda_{12}=\frac{1}{t}-\frac{1}{p T}, \lambda_{13}=\frac{1}{p T}$, and $\lambda_{21}=\frac{1}{t}$, where $p=\frac{t}{t+t}$ is the node availability. Finally, we assume that nodes are homogeneous.

Replication and repair model The system uses a combination of replication and repair to implement durable storage. The process of replication consists of making copies of an object; the system is said to create a replica when it copies the object onto some node in the system. The degree of replication is defined by the number of replicas of the object initially created by the system; we denote this system parameter by $r$.

Given a degree of replication $r$, the system starts by creating $r$ replicas of the object. Subsequently, the system attempts to maintain $r$ replicas of the object at all times through a process of repair. Repair is necessary to replace replicas that are dead; otherwise, node departures will eventually result in the permanent loss of the object when all its replicas are dead. To prevent this, the system must effect a repair, i.e., create a new replica of the object whenever it detects that an existing replica is dead. Ideally, a repair should be made only when a replica is dead; however, the temporary unavailability of an offline replica introduces several subtleties in the repair process.

First, the system cannot distinguish between a replica that is dead, and one that is merely offline. Thus, the system cannot reliably detect when a replica is dead; each time a replica transitions out of the online state, the system is dealing with the potential loss of a replica, and must decide whether to make a repair or not. We assume that the system uses a timeout mechanism to determine when to trigger a repair, i.e., it waits some period of time for the replica to return to the online state. If the replica returns before the timeout occurs, no repair is necessary; on the other hand, if it does not, the system assumes that it is dead, and effects a repair.

Choosing a timeout value depends on the balance between causing frequent, and perhaps unnecessary, repairs when the system prematurely times out a replica (i.e., one that would return if the system waited longer), and delaying replacing a replica when it actually is dead. It is natural to express the timeout period in terms of mean node downtime since 


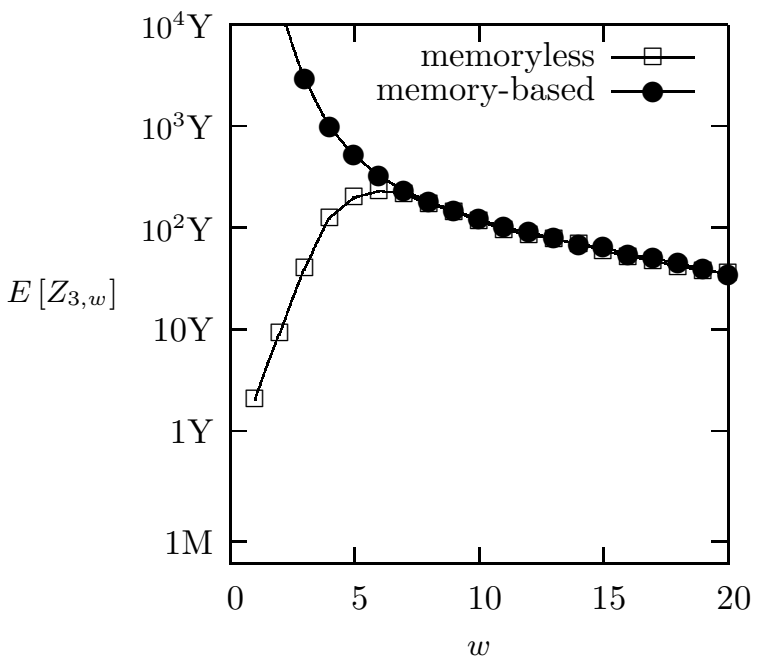

(a) Mean data lifetime

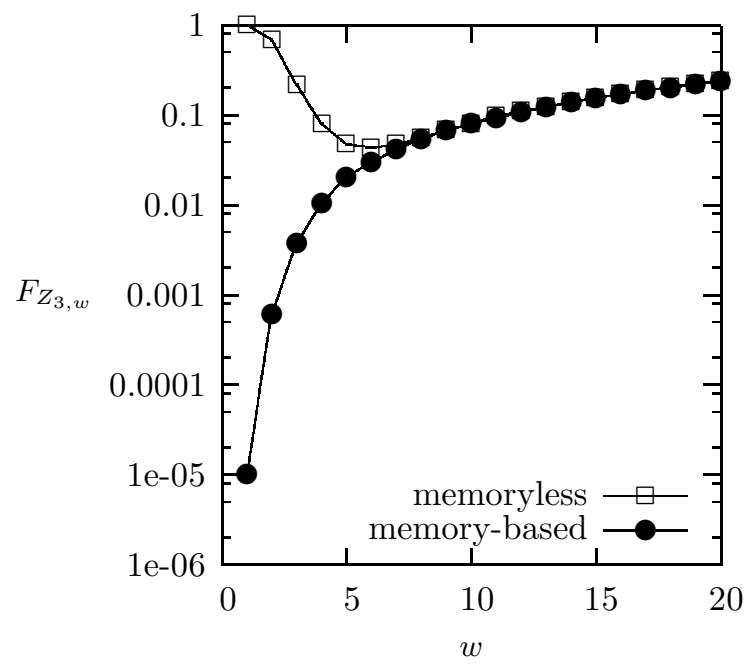

(b) Probability that data lifetime is less than 10 years

Figure 1: Data lifetimes for $r=3$ replicas with node parameters $T=1$ year, $t=159.5$ hours, and $\bar{t}=49.6$ hours. Uptimes and downtimes are derived from PlanetLab data.

on average, a replica is offline for a period of $\bar{t}$. Thus, we assume the timeout is given by $w \bar{t}$, where $w$ is a system parameter. A key question is, is there an optimal value for $w ?$

Second, the timeout mechanism is not perfect; the system will occasionally time out a replica that is not dead, but is merely offline for a duration exceeding the timeout value. Repair is said to be memoryless if the system simply discards such a replica, i.e., it does not reintegrate this replica into the set of remaining ones. Rather, it assumes that the replica is lost, in effect, treating that node as though it were joining the system for the first time. Alternatively, repair is said to be memory-based, if the system readmits the timedout replica to the set of existing replicas, taking advantage of what is essentially a "free" repair. Memoryless repair is simpler than memory-based repair, as the system does not need to "remember" timed-out replicas, but is also potentially more inefficient. A key question is, how significant is this inefficiency, and can it be tolerated to enjoy the benefits of design and implementation simplicity?

\section{RESULTS}

We use Monte Carlo simulation of our system model to derive data lifetime, i.e, the time that elapses between the instant the system initially replicates an object, and the instant the system no longer has any replicas of that object. Figure 1 presents data lifetime as a function of timeout $w$ for $r=3$ replicas in the case of both memoryless and memorybased repair; we consider both mean lifetimes and 10 year loss probabilities.

Our first result is that while the durability of memorybased repair is monotonic w.r.t. $w$, as evidenced by decreasing mean lifetime and increasing loss probability, this is not true for memoryless repair. Somewhat counter-intuitively, it is not the case that smaller $w$ results in greater durability; rather, there exists an optimal value of $w$, occurring at $w \approx 6$ in the graphs of Figure 1 , at which mean lifetime is maximum, and loss probability is minimum. This behavior can be explained by the fundamental inefficiency of memoryless repair; without memory, the system discards replicas it erroneously timed out when they return, even though retaining them would clearly be useful. Decreasing the timeout increases the frequency of such "mistakes", and beyond a point, the penalty associated with prematurely timing out replicas that are only offline outweighs the ability to quickly replace a replica that is actually dead. In ongoing work [4], we study the sensitivity of the optimal value of $w$ w.r.t. node parameters $T, t$ and $\bar{t}$.

Our second result is that while memory-based repair is superior when $w$ is small, for larger values of $w$, data lifetimes are comparable, as can be seen in the graphs of Figure 1. This implies that, beyond some threshold value of $w$, there is a regime in which memoryless repair is indeed a viable alternative to the more complex memory-based repair. When we additionally incorporate the notion of cost, defined as replicas created by the repair process per unit time, we find that the benefits of memory can be fully realized only if the system can support a sharply higher cost of repair [4]. This is because a smaller $w$ increases the frequency of spurious timeouts, and hence repairs.

\section{REFERENCES}

[1] Adya, A., Bolosky, W., Castro, M., Cermak, G., Chaiken, R., Douceur, J., Howell, J., Lorch, J., Theimer, M., And WattenHOFER, R. Farsite: Federated, available, and reliable storage for an incompletely trusted environment. In Proceedings of USENIX OSDI (2002).

[2] Chun, B., Dabek, F., Haeberlen, A., Sit, E., Weatherspoon, H., KaAshoek, F., Kubiatowicz, J., And Morris, R. Efficient replica maintenance for distributed storage systems. In Proceedings of USENIX NSDI (2006).

[3] Dabek, F., Kanshoek, F., Karger, D., Morris, R., and Stoica, I. Wide-area cooperative storage with cfs. In Proceedings of $A C M$ SOSP (2001).

[4] Ramabhadran, S., and Pasquale, J. Analysis of the durability of replicated distributed storage systems. UCSD Technical Report CS2007-0900 (2007).

[5] Rowstron, A., And Druschel, P. Storage management and caching in past, a large-scale, persistent peer-to-peer storage utility. In Proceedings of ACM SOSP (2001). 